\title{
Steps to Getting Your Manuscript Published in a High-Quality Medical Journal
}

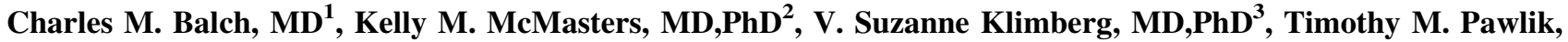 \\ MD,MPH,PhD ${ }^{4}$, Mitchell C. Posner, $\mathrm{MD}^{5}$, Mark Roh, $\mathrm{MD}^{6}$, Kenneth K. Tanabe, $\mathrm{MD}^{7}$, Deborah Whippen, $\mathrm{BA}^{8}$, and \\ Naruhiko Ikoma, MD,MS
}

\begin{abstract}
${ }^{1}$ Department of Surgical Oncology, The University of Texas MD Anderson Cancer Center, Houston, TX; ${ }^{2}$ Department of Surgery, University of Louisville, Louisville, KY; ${ }^{3}$ Division of Surgical Oncology, Department of Surgery, University of Texas Medical Branch, Galveston, TX; ${ }^{4}$ Department of Surgery, Ohio State University, Columbus, OH; ${ }^{5}$ Department of Surgery, University of Chicago, Chicago, IL; ${ }^{6} \mathrm{UF}$ Health Cancer Center, Orlando Health, Orlando, FL; ${ }^{7}$ Division of Surgical Oncology, Massachusetts General Hospital Cancer Center and Harvard Medical School, Boston, MA; ${ }^{8}$ Editorial Office, Annals of Surgical Oncology, North Fort Myers, FL
\end{abstract}

\begin{abstract}
Publication of your research represents the culmination of your scientific activities. The key to getting manuscripts accepted is to make them understandable and informative so that your colleagues will read and benefit from them. We describe key criteria for acceptance of manuscripts and outline a multi-step process for writing the manuscript. The likelihood that a manuscript will be accepted by a major journal is significantly increased if the manuscript is written in polished and fluent scientific English. Although scientific quality is the most important consideration, clear and concise writing often makes the difference between acceptance and rejection. As with any skill, efficient writing of high-quality manuscripts comes with experience and repetition. It is very uncommon for a manuscript to be accepted as submitted to a journal. Thoughtful and respectful responses to the journal reviewers' comments are critical. Success in scientific writing, as in surgery, is dependent on effort, repetition, and commitment. The transfer of knowledge through a well-written publication in a high-quality medical journal will have an impact not only in your own institution and country, but also throughout the world.
\end{abstract}

(C) Society of Surgical Oncology 2018

First Received: 22 September 2017;

Published Online: 18 January 2018

C. M. Balch, MD

e-mail: CMBalch@mdanderson.org
Publication of a scientific manuscript is the ultimate and arguably one of the most important components of research. ${ }^{1}$ Through publication of a scientific manuscript, your knowledge and experience become citable science in the form of novel information transmitted to physicians and scientists for current and future generations. ${ }^{2}$

Writing manuscripts can be a rewarding and satisfying creative process, and publication of manuscripts confers substantial benefits to academic surgeons. ${ }^{3}$ Publications in high-quality journals lend credibility to your research (due to the rigorous peer review of high-quality journals and the intense competition for the limited publication space in such journals) and are highly valued evidence of scholarship by promotion and tenure committees. A strong track record of publications carries significant influence in the allocation of grants.

For trainees and surgeons early in their careers, writing manuscripts can be a valuable learning experience. As with any skill, efficient writing of high-quality manuscripts comes with experience and repetition. In this article, we offer suggestions to speed the learning process for trainees and early-career surgeons. This advice is relevant not only for manuscripts submitted to Annals of Surgical Oncology, for which most of us serve as editorial leaders, but also for clinical research manuscripts in general.

\section{GOALS OF PUBLICATION}

Publications in high-quality journals are important to communicate your research findings and tell your "story" about the new information you have gained from your 
research efforts based on a novel question or hypothesis. Publication of your findings is most valuable when your colleagues can find your article in the medical literature (your title and keywords are critical), understand your paper and believe the results (the key points in the Results and Discussion sections), recognize the ramification of the results and the unique contributions of your research (hypothesis and results are both impactful and original), incorporate your data into their own fund of knowledge, and quote or cite your paper in their own publications (the ultimate metric of your publication impact in the biomedical literature). Before you begin writing your manuscript, consider how you will craft the manuscript to achieve these outcomes.

\section{MANUSCRIPT EVALUATION CRITERIA}

As you write and revise your manuscript, keep in mind key criteria for acceptance of manuscripts (Table 1). These criteria help reviewers determine whether the research

TABLE 1 Criteria for acceptance of manuscripts

Significance does it pass the "so what?" test?
Originality of the research and conclusions
Methodologic and statistical validity
Ethical research practices
Impact on clinical practice or on science
Article citable by other authors?
Clarity of message and data presentation
Appropriateness or relevance of the subject for the journal's
readership

Significance does it pass the "so what?" test?

Originality of the research and conclusions

Methodologic and statistical validity

Ethical research practices

Impact on clinical practice or on science

Clarity of message and data presentation

readership

TABLE 2 Common reasons for rejection of manuscripts

General Topic not of sufficient interest to readership, manuscript does not conform to journal requirements, subject not appropriate for journal readership

Methods Retrospective design, case study, small sample size, follow-up too short, or invalid statistics or control

Results Nothing new, data previously presented elsewhere, data not supportive of conclusions, or outcomes worse than those with the current standard of care

Discussion section Poorly written (grammar/content), conclusions vague or not supported by the data, found in another of your manuscripts, or references incomplete

TABLE 3 Multi-step process for getting manuscripts accepted findings are of importance and the manuscript is appropriate for the journal. The leading clinical journals primarily seek manuscripts that have an immediate impact on clinical practice because others will cite such manuscripts and increase the journal's impact and reach.

Select research topics to address key clinical questions. Then, conduct a valid and reliable study and analyze the results with appropriate statistical methods. Be sure that your study is different from previously reported studies (e.g., not just longer follow-up period or addition of 30 more patients), and do not divide the results of a single study into multiple publications unless those publications are complementary, not duplicative. Common reasons for rejection of manuscripts are summarized in Table 2 and also have been described in other papers. ${ }^{4,5}$

\section{A MULTI-STEP PROCESS FOR GETTING MANUSCRIPTS ACCEPTED}

One way to organize your efforts to get your manuscript accepted is to follow the multi-step process outlined in Table 3. To ensure the most accurate text, we recommend writing the manuscript shortly after you finalize your results.

\section{Step 1: Confirm Authors, Select a Journal, and Read} the Journal's Instructions

Deciding in advance on authorship as well as the sequence of authors, including the corresponding author, is important to avoid conflict. ${ }^{6}$ All authors listed on the final manuscript must have participated sufficiently in the work to be accountable and take public responsibility for the

Step 1: Confirm authors, select a journal, and read the journal's instructions

Step 2: Prepare the manuscript; ensure that all authors contribute to and approve of the final version

Step 3: Submit the manuscript with all authors' conflicts of interest disclosed in the manuscript

Step 4: Receive the editors' communication and revise the manuscript

Step 5: Resubmit revised manuscript with changes highlighted and a summary letter describing responses to reviewers' comments 
content of the publication. ${ }^{7}$ The International Committee of Medical Journal Editors lists four criteria for authorship: (1) "substantial contributions to the conception or design of the work, or the acquisition, analysis, or interpretation of data for the work" AND (2) "drafting the work or revising it critically for important intellectual content" AND (3) "final approval of the version to be published" AND (4) "agreement to be accountable for all aspects of the work in ensuring that questions related to the accuracy or integrity of any part of the work are appropriately investigated and resolved." "Courtesy authorship" is unacceptable and unethical and should be avoided. ${ }^{9}$

After conducting quality research, select an appropriate journal for submission of your work, with an appropriate target readership and impact factor. ${ }^{9}$ Matching the content, quality, and impact of your research to the appropriate journal will improve your manuscript's chance of acceptance and reduce the frustration of multiple rejections. ${ }^{10}$ Annals of Surgical Oncology (ASO), with its current 5-year impact factor of 4.308, often is the journal of choice for high-quality manuscripts in the field of surgical oncology.

When preparing your manuscript, carefully follow the journal's requirements, including instructions regarding the word limit and limits on the numbers of tables and figures. The word count limit for ASO is 2500 , excluding the title page, abstract, and references. Only five figures and/or tables are allowed per manuscript. Therefore, plan ahead for the total number of tables and figures needed to make your key points. Many journals, including ASO, allow for supplementary material to be submitted as part of your manuscript, exclusive of the word count or figures/ tables limits.

\section{Step 2: Prepare the Manuscript}

Several strategies have been proposed for writing scientific manuscripts. ${ }^{3,11,12}$ One strategy is to write the Methods section, then the Results, Discussion, and Introduction sections, in that order. The easiest section of a manuscript to write typically is the Methods section. After all, the author knows how the study was designed from the very beginning and can easily draft a Methods section with appropriate subheadings. The subheadings in the Results section often can directly mirror the subheadings in the Methods section (e.g., Study Population, Pathology Results, Radiologic Results). The Discussion section, including a conclusion/summary statement, naturally follows from the Results section. The Introduction section often is written last. Use of modern reference management software allows easy insertion of references as you write and allows references to be moved easily to various sections as needed and renumbered automatically.
An alternative writing strategy, used by many experienced scientific writers, is first to create a draft layout of the figures and tables in the desired order and then to write the figure legends. The optimal sequence for presentation of data and figures is not necessarily the order in which experiments were performed. Rather, the sequence that provides for the clearest presentation should be used. After creation of the figures, the text of the Results section is written so that it is supplemented by the figures and tables (e.g., not repetitive). The other sections are written next, with drafting of the abstract postponed until drafts of the other sections are complete.

Each author should be familiarize him- or herself with the scientific writing for a variety of study designs (e.g., STROBE for observational studies, PRISMA for systemic reviews and meta-analyses, and CONSORT for randomized control trials). ${ }^{13}$

Outline the Manuscript Clinical research manuscripts need to be well structured. The scientific manuscript structure followed by most clinical journals is summarized in Table 4.

Liberal use of subheadings, especially in the Methods and Results sections, is often useful to the reader. Avoid placing contents in the wrong sections, which can confuse the reviewers. For example, do not describe partial results in the Methods section, and avoid speculation regarding data in the Results section. Such fundamental flaws are quickly detected by reviewers and often result in a request for revision or rejection of the manuscript.

With this basic structure in mind, begin by outlining the manuscript. The very first primitive draft would be a list of important key points you want to describe under each section and subheading. First, make sure your thoughts are expressed. Then determine whether you have expressed them in the correct section and in a logical sequence. This procedure will provide you with a rough draft of the manuscript, maintain your focus on important points, and avoid sloppy and lengthy manuscripts.

Draft the Manuscript Once the primitive outline is complete, develop a single, coherent manuscript that logically explains your study. It is important to focus around a hypothesis and to ensure that the entire Results section, the Discussion section, and the title consistently address the stated hypothesis. The conclusion of the manuscript should directly align with the data presented in the Results section, and overstatement must be avoided.

For academic surgeons, it is very important to be a student of fluent writing in English. Scientific writing requires specialized writing skills that are not well emphasized in medical education in general or research training in particular. The likelihood that a manuscript will 
TABLE 4 Common structure of clinical manuscripts

Title Write an informative title that will capture the reader's interest. Titles should be a precise summary of the paper's content, brief and clear. Avoid jargon and abbreviations

Abstract The abstract is the part that will be read first and most widely. Follow the journal format and word length for the abstract (typically about 250 words), and be sure to include the key data and conclusions

Key words (6-8) Key words help readers find your paper and support its listing to be at the top in search results. Avoid overlapping key words as well as key words that are only one word

Introduction The Introduction section should include 2 to 3 paragraphs about the background summarizing existing evidence and knowledge gaps, purpose of the study, and hypothesis (if the study was hypothesis driven) to convince readers that the study is significant and important

Methods Provide enough details (or citations if similar methods were reported in previous studies) so that others can judge the reliability of the study and reproduce your research. Clearly document the study design, study subjects, time period, metrics and outcomes, and statistical methods

Results Present the data and results germane to the hypothesis or the theme of the manuscript. Appropriate use of tables and figures is critical. Avoid repetition in the text of data presented in tables and/or figures. You may describe your objective interpretation of the data in the Results section rather than simply listing observations

Discussion Summarize the study and explain both the significance of the findings and how they fit in with previously published work. In other words, explain "what the findings mean." We recommend writing a Discussion section that includes 5 paragraphs. In the first paragraph, briefly summarize the study results, their significance, and the originality of the findings. In the second paragraph, describe your study's relationship to other studies. In the third paragraph, discuss specific findings of your choice (e.g., speculate about unexpected findings). In the fourth paragraph, describe the limitations of the study. In the final paragraph, briefly summarize the global conclusions, carefully avoiding overstatement of the study results. Although it is challenging, avoid excessive repetition of results between the Results section, Figures, and Discussion section

References List the publications cited in the manuscript. Use of citation software such as Endnote to insert and format the references is strongly recommended

Figure legends Write the legends such that they are brief and make the figures fully understandable without reference to the text of the manuscript

be accepted by a major journal is significantly increased if the manuscript is written in polished and fluent scientific English. Particularly for authors who are not native English speakers, having the manuscript reviewed by a scientific editor before manuscript submission often is of substantial benefit. ${ }^{59}$ English editing is not the journal's responsibility. Well-written English will surely give the reviewer a more favorable impression of the manuscript.

Brevity without sacrifice of logic and clarity is a cardinal rule in scientific publishing. ${ }^{5}$ A concise manuscript without redundancy not only helps readers understand, but also reduces the cost of publication. Present your data efficiently, logically, and clearly focused on the study's main purpose or objective.

Effective tables and figures are of paramount importance to the presentation of findings ${ }^{14}$ and also are extremely helpful in making the manuscript concise. In addition, electronic files, especially videos, often are useful and favored tools that strengthen the manuscript. At ASO, articles including videos are encouraged and can be submitted as Multimedia Articles [papers that include video (9 min at the maximum) with an accompanying abstract and references] or Dynamic Articles [regular articles that include a supplementary video (up to 3 video clips, $3 \mathrm{~min}$ at the maximum for each)].

In drafting the manuscript, pay utmost attention to avoid plagiarism (including self-plagiarism), fraud, and fabrication. All authors of a manuscript are responsible for any such infractions that are committed. ${ }^{15}$ Disclosure of conflicts of interest has been standardized across most clinical journals, and authors must acknowledge all support received from industry for their research, regardless whether the funds were received directly or indirectly.

Obtain Feedback from Colleagues and Revise the Manuscript After drafting the manuscript, distribute your draft to co-authors and one or two non-author colleagues to gain their feedback and their "collective wisdom." After revising to ensure that your idea or ideas have been correctly and fully expressed, address and correct the English. The more iterations of the manuscript you have, with critical input from co-authors, the better.

\section{Step 3: Submit the Manuscript}

Most scientific journals now require submission through their websites. During manuscript submission, most journals allow authors to suggest reviewers who should and should not review an article, and such suggestions are helpful to the journals' editorial staffs. Carefully choose the title and keywords so readers can find your paper. A concise cover letter, including a summary of the manuscript's significance in two to three sentences, can provide important context to the editor. ${ }^{16}$ DO NOT submit the same 
manuscript simultaneously to two journals because doing so wastes resources and is a serious violation of scientific publishing ethics. ${ }^{17}$

At ASO, manuscripts are received electronically (about 60 per week), with an average time of 28 days to the first decision. ${ }^{18}$ For most submissions, the Section Editor or Associate Section Editor assigns a minimum of two reviewers per manuscript. Their comments and recommendations, together with those of the Section Editor, are passed to the Executive Editor for a final decision. The corresponding author is notified of acceptance (with or without minor revisions), rejection with advice to resubmit, or rejection.

\section{Step 4: Receive the Editor's Communication and Revise the Manuscript}

Acceptance of a manuscript as submitted is very uncommon. If your manuscript is rejected, do not feel insulted! In fact, fewer than one in four manuscripts are accepted by top journals. If revisions are invited, submission of a careful and respectful response is of paramount importance to increase the probability of subsequent acceptance. ${ }^{19-21}$ It is good to remember that revisions invariably make the manuscript better. In rare instances, a reviewer's comments may not be germane or acceptable. If you feel a specific revision is unjustified, state why in an unemotional and straightforward manner in the response letter. ${ }^{21}$ Realize, however, that most reviewers and editorial board members of top journals are experienced experts and therefore rebut reviewer suggestions infrequently, carefully, and in a respectful manner.

\section{Step 5: Resubmit the Manuscript}

When you submit a revised manuscript, include a cover letter to the editor with point-by-point responses to the reviewers' comments and suggestions, and try to address all criticisms and suggestions as fully as possible. Highlight changes in the revised manuscript to allow for careful review. If requested data are not available, it is appropriate to state that this is the case. Prompt response in a reasonable time frame (ideally within less than a month) is strongly encouraged. You may not be able to make all the changes suggested by all of the reviewers. Nevertheless, a respectful, thorough, good faith effort to address the suggestions as completely as possible, and, if applicable, a clear and reasonable explanation why some changes were not possible often will result in acceptance.

\section{WRITE FROM A REVIEWER'S PERSPECTIVE}

The review process is an educational process for authors. The more scientific manuscripts you submit (and revise), the better writer you will become. To enhance your scientific writing skills, find opportunities to become a reviewer for top journals and/or a member of their editorial boards. Although being a journal reviewer is time-consuming, it has significant benefits for your career. ${ }^{22}$ Reviewing other manuscripts keeps you updated on topics in the field. Critical thinking about manuscripts helps you polish your own writing skills. You also will have a valuable learning opportunity to review other reviewers' comments on the same manuscript. After contributing several critical and on-time reviews for the same journal, it is reasonable to write the editorial office with your curriculum vitae and ask to become a member of the editorial board, which provides you a credential as an academic researcher.

\section{CONCLUSIONS}

Publication of your research represents the culmination of your scientific activities. The key to getting manuscripts accepted is to make them understandable and informative so that your colleagues will read and benefit from your manuscripts. Although scientific quality is the most important consideration, clear and concise writing often makes the difference between acceptance and rejection. Thoughtful and respectful responses to reviewers' comments are critical. Success in scientific writing, as in surgery, is more dependent on effort, repetition, and commitment than on innate talent. Enjoy writing and recognize that your publications may have an impact not only in your own institution and country, but also throughout the world.

ACKNOWLEDGMENT We thank Ms. Stephanie Deming, Senior Scientific Editor, Department of Scientific Publications at UT MD Anderson Cancer center, for her editorial input.

DISCLOSURE There are no conflicts of interest.

\section{REFERENCES}

1. Kotz D, Cals JW, Tugwell P, Knottnerus JA. Introducing a new series on effective writing and publishing of scientific papers. $J$ Clin Epidemiol. 2013;66:359-60.

2. Knottnerus JA, Tugwell P. Communicating research to the peers. J Clin Epidemiol. 2007;60:645-47.

3. Kibbe MR. How to write a paper. ANZ J Surg. 2013;83:90-92.

4. Audisio RA, Stahel RA, Aapro MS, Costa A, Pandey M, Pavlidis N. Successful publishing: how to get your paper accepted. Surg Oncol. 2009;18:350-56. 
5. Chernick V. How to get your paper accepted for publication. Paediatr Respir Rev. 2012;13:130-32.

6. Cals JW, Kotz D. Effective writing and publishing scientific papers, part IX: authorship. J Clin Epidemiol. 2013;66:1319.

7. Bosch X, Pericas JM, Hernandez C, Torrents A. A comparison of authorship policies at top-ranked peer-reviewed biomedical journals. Arch Intern Med. 2012;172:70-72.

8. International Committee of Medical Journal Editors. Defining the Role of Authors and Contributors. Retrieved 11 September 2017 at http://www.icmje.org/recommendations/browse/roles-andresponsibilities/defining-the-role-of-authors-and-contributors. html\#two.

9. Kibbe MR, Sarr MG, Livingston EH, Freischlag JA, Lillemoe $\mathrm{KD}, \mathrm{McF}$ adden DW. The art and science of publishing: reflections from editors of surgery journals. J Surg Res. 2014;186:7-15.

10. Cals JW, Kotz D. Effective writing and publishing scientific papers, part X: choice of journal. J Clin Epidemiol. 2014;67:3.

11. Johnson TM. Tips on how to write a paper. J Am Acad Dermatol. 2008;59:1064-69.

12. Kotz D, Cals JW. Effective writing and publishing scientific papers, part I: how to get started. J Clin Epidemiol. 2013;66:397.

13. NIH, U.S. National Library of Medicine website. Research Reporting Guidelines and Initiatives: By Organization. Retrieved 18 August at https://www.nlm.nih.gov/services/research_report_ guide.html.
14. Kotz D, Cals JW. Effective writing and publishing scientific papers, part VII: tables and figures. $J$ Clin Epidemiol. 2013;66:1197.

15. Bosch X, Hernandez C, Pericas JM, Doti P, Marusic A. Misconduct policies in high-impact biomedical journals. PLoS One. 2012;7:e51928.

16. Kotz D, Cals JW. Effective writing and publishing scientific papers, part XI: submitting a paper. J Clin Epidemiol. 2014;67:123.

17. Balch CM. Duplicate submission and scientific fraud: the Annals of Surgical Oncology perspective. Ann Surg Oncol. 2001;8:381.

18. Balch CM, Klimberg VS, McMasters KM, et al. Benchmarking the scientific and educational impact of the Annals of Surgical Oncology. Ann Surg Oncol. 2016;23:2723-39.

19. Haskal ZJ, Coler E. How to get your paper published in JVIR. $J$ Vasc Interv Radiol. 2015;26:1456-58.

20. Sullivan GM. What to do when your paper is rejected. J Grad Med Educ. 2015;7:1-3.

21. Kotz D, Cals JW. Effective writing and publishing scientific papers, part XII: responding to reviewers. J Clin Epidemiol. 2014;67:243.

22. Stahel PF, Moore EE. How to review a surgical paper: a guide for junior referees. BMC Med. 2016;14:29. 